\title{
Design Aspects of X-Ray Grating Interferometry
}

\author{
Timm Weitkamp*, Irene Zanette ${ }^{\dagger}$, Franz Pfeiffer ${ }^{\dagger}$ and Christian David** \\ ${ }^{*}$ Synchrotron Soleil, Gif-sur-Yvette, France \\ ${ }^{\dagger}$ Department of Physics, Technische Universität München, Garching, Germany \\ ${ }^{* *}$ Laboratory for Micro- and Nanotechnology, Paul Scherrer Institut, Villigen, Switzerland
}

\begin{abstract}
Considerations are made for the design of X-ray grating interferometers in general and, in particular, for the case of a parallel beam with a high degree of spatial coherence. We specifically discuss the properties of different types of gratings and the interdependence of instrument parameters and performance characteristics.
\end{abstract}

Keywords: X-ray phase contrast imaging; Talbot interferometry; phase-shifting interferometry; microtomography; synchrotron radiation; X-ray optics

PACS: $87.59 .-\mathrm{e}, 42.30 . \mathrm{Va}, 42.79 . \mathrm{Dj}, 42.30 . \mathrm{Ms}$, 42.87.Bg, 42.25.Fx

\section{INTRODUCTION}

X-ray grating interferometry (XGI) was first demonstrated on synchrotron sources [1-8]. Today, while the implementation of XGI with X-ray tube sources is rapidly progressing, this technique continues to gain importance at synchrotron imaging beamlines, both because of the high density resolution that XGI phase imaging offers and because XGI gives access to multiple imaging modes, including "dark-field" imaging [9], also referred to as "visibility contrast" [10]. While applications in beam diagnostics and Xray optics characterization exist [11, 12], most of the fixed setups are optimized for imaging applications, and particularly for phase tomography [13-17].

Figure 1 (left) shows the schematic setup of an XGI as first realized by Momose [2]. This device, also known as a Talbot interferometer, consists of two transmission gratings. Instruments of this type are today available at several synchrotron imaging beamlines [18-20]. A third grating can be added to increase the efficiency when an XGI is used at a source of low brilliance [21]. The three-grating setup is usually named a Talbot-Lau interferometer [22]. While it is most often used with laboratory sources, it can also be beneficial at synchrotron sources of limited brilliance, for example at second-generation light sources [23]. The scope of the present article is limited to the two-grating XGI.

There are several important figures of merit for an X-ray grating interferometer, in particular the resolution in refraction angle and the spatial resolution of the instrument. These are are related to instrument parameters such as

- the pitch of the gratings used;

- the length of the instrument, i.e., the distance between G1 and G2;

- the sensitivity of the instrument, i.e., the ratio between observed fringe phase and wavefront propagation direction.

- the visibility of the fringes observed in moiré mode or in the intensity oscillations in a phase stepping scan, which has a critical influence on the signal-to-noise ratio

International Workshop on X-ray and Neutron Phase Imaging with Gratings

AIP Conf. Proc. 1466, 84-89 (2012); doi: 10.1063/1.4742273

(C) 2012 American Institute of Physics 978-0-7354-1072-5/\$30.00 

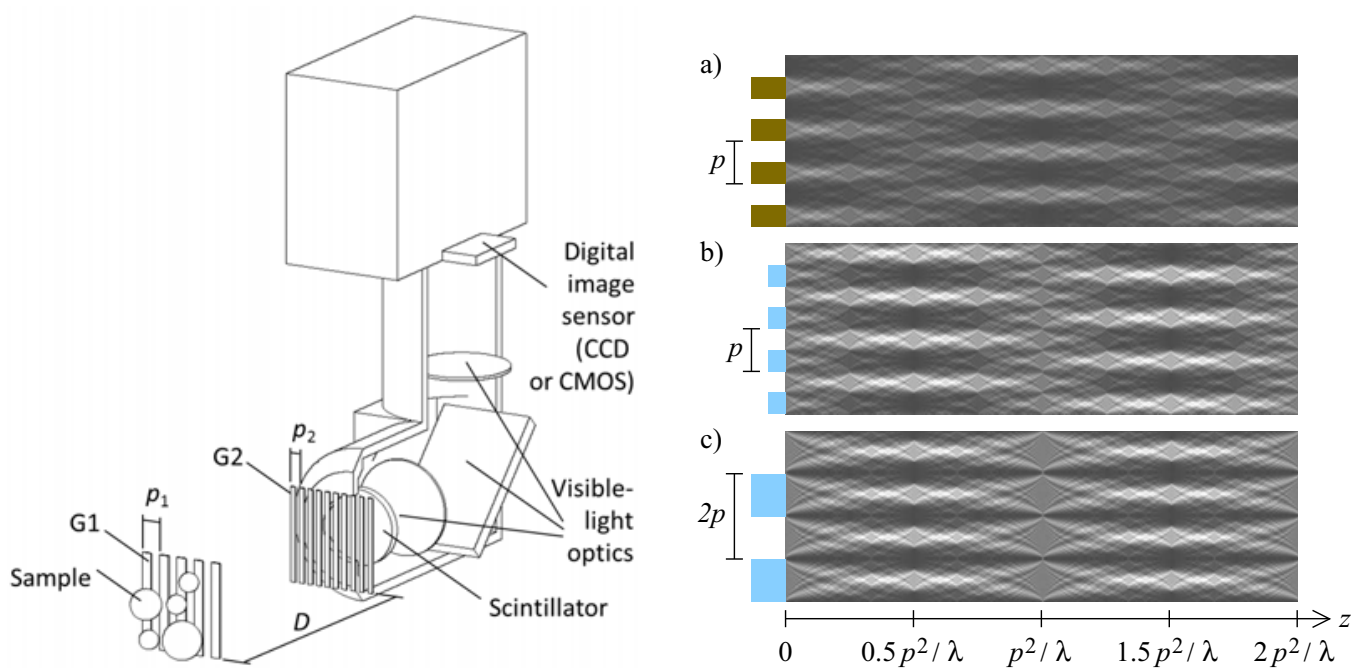

FIGURE 1. Left: Schematic of a typical XGI setup used in high-resolution synchrotron microtomography. The two-grating interferometer, consisting of a beam-splitter grating G1 with pitch $p_{1}$ and an analyzer grating $\mathrm{G} 2$ with pitch $p_{2}$, separated by a distance $D$, is placed between the sample and a high-resolution $\mathrm{X}$-ray imaging detector, here a system consisting of a scintillator that creates a visible-light image which is then projected onto the sensor of a digital detector. Elements not shown in this figure include the Xray source (bending magnet, wiggler or undulator), the X-ray optics used for beam transport and beam conditioning, and the motion stages used for alignment and scanning. Right: Simulated intensity patterns behind different types of gratings G1 that yield the same fringe spacing $p$. (a) Absorption grating, pitch $p_{1}=p$. (b) $\pi / 2$-shifting phase grating, pitch $p_{1}=p$. (c) $\pi$-shifting phase grating, pitch $p_{1}=2 p$.

and thus on the efficiency of the device;

- the coherence length of the illuminating radiation.

In the following, we will discuss the influence of some design parameters on these figures, and their interdependence.

\section{GRATING DESIGN}

Optical properties. To achieve optimum visibility, while the absorbing structures of the analyzer grating $\mathrm{G} 2$ should have a transmission as close to zero as possible, the beam splitter G1 should ideally either also have completely absorbing lines, or its lines should be completely transparent but shift the X-ray phase by either $\pi / 2$ (i.e., by a quarter of a wavelength) or by $\pi$ (i.e., by half the wavelength) $[2,24]$. A phase grating is obviously the more photon-efficient choice for G1 than an absorption grating.

Moreover, as is well known, the Talbot effect causes a periodic variation of the visibility as a function of distance between the beam splitter grating G1 and the analyzer G2. At synchrotron beamlines, the beam can be considered parallel for most though not all purposes, and in the following all considerations presume an incident plane wave unless otherwise specified. In particular, the distances behind G1 at which the fringe 


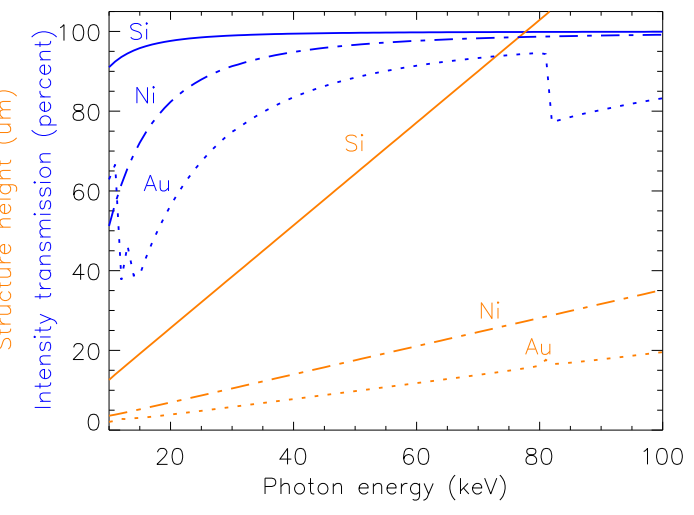

FIGURE 2. Intensity transmission (blue) through the lines of a $\pi$-shifting phase grating and required structure depth of the grating (orange) for three different materials, as a function of X-ray photon energy. Low- $Z$ elements have good transmission but require deep lines.

visibility takes extreme values are, in the plane-wave case [24],

$$
D_{n}=n \frac{p_{1}^{2}}{2 \eta^{2} \lambda}=n \frac{p_{2}^{2}}{2 \lambda}
$$

Here, $n$ is an integer number often referred to as the fractional Talbot order, $p_{1}$ and $p_{2}$ are the respective periods of G1 and G2, and $\lambda$ is the X-ray wavelength. If G1 is an absorption grating, then the visibility maxima correspond to $n=0,2,4, \ldots$; if it is a phase grating, they are observed at odd values of $n$. The parameter $\eta$ is a dimensionless number describing the grating: if G1 is an absorption grating or a phase grating with $\pi / 2$-shifting lines, then $\eta=1$; for a $\pi$-shifting phase grating, $\eta=2$.

The right part of Fig. 1 shows the intensity distributions after G1 for each of these types of grating. In the following we mention some implications of the behavior described by the equations above and illustrated in the figure.

If G1 is an absorption grating, then the first visibility maximum is obviously $D_{1}=0$, i.e., the contact plane behind G1. This means that in this case it is possible to place G2 in a plane located at a distance $D<p_{2} / \lambda$, i.e., in the near field of G1. Such a device operates in the same regime as a Hartmann sensor [25]. Grating-based imaging techniques based on this principle exist $[26,27]$ but are not commonly implemented on synchrotron stations.

If $\mathrm{G} 1$ is an absorption grating or a $\pi / 2$-shifting phase grating, the contrast is inversed between each pair $D_{n}$ and $D_{n+2}$ of adjacent contrast maxima, but this is not the case for a $\pi$-shifting phase grating. This feature of $\pi$-shifting gratings is particularly useful when using grating interferometry with broadband radiation, where it allows taking advantage of the panchromatic effect [28, 29].

For fixed fringe spacing $p_{2}$, the aspect ratio of a phase-shifting G1, i. e., the ratio of the grating's structure depth to its period, does not depend on whether G1 is a $\pi / 2$-shifting or a $\pi$-shifting grating. Both the pitch $p_{1}$ of G1 and the structure height $h_{1}$ of its lines need to be twice as large for a $\pi$-shifting grating as for a $\pi / 2$ grating. 
The previous point implies that unwanted absorption from the grating lines is stronger in a $\pi$ grating than in a $\pi / 2$ grating for the same photon energy.

The optimum grating parameters for an interferometer will therefore depend on many parameters including the photon energy, spectral bandwidth of the radation, available fabrication technology, etc.

Grating material. When choosing the grating material for a G1 phase grating, two parameters should be optimized: on the one hand, the absorption in the grating lines should be kept minimal - not only because absorption in the gratings reduces the photon efficiency of the device, but also because the Talbot distances and/or fringe profiles change with respect to the case of the ideal phase grating when absorption is present. On the other hand, the material must be chosen so that the depth and aspect ratio of the structures remain within technologically feasible limits.

Unfortunately, these two requirements are effectively contradictory, as Fig. 2 shows. Dense materials made of heavy elements have a relatively poor ratio between phase shift and absorption, whereas the use of lighter elements, where this ratio is more favorable, requires the fabrication of very deep structures. Therefore, in practice, a wide range of materials and atomic numbers from light to heavy elements is actually used in G1 gratings of XGI implementations around the world. For example, at the ESRF beamline ID19 [19, 30], $\pi$-shifting silicon gratings [31] are used as beam splitters for energies up to $35 \mathrm{keV}$, and $\pi / 2$-shifting nickel or gold gratings [32] for higher energies.

In addition to the periodic visibility oscillation described by Equation 1, the visibility observed in the maxima decreases with increasing Talbot order $n$ because of the limited spatial coherence of the X-ray beam. The transverse coherence length at the position of G1 that is required to obtain $80 \%$ of the maximum visibility is [24]

$$
\ell_{80 \%}=2 n p_{2} \text {. }
$$

\section{SENSITIVITY AND PERFORMANCE CHARACTERISTICS}

As mentioned above, the sensitivity is one of the fundamental parameters of a grating interferometer. In this section we will relate this quantity to some of the performance characteristics of the interferometer. We follow Donath et al. [33] who, in accordance with terminology rules in metrology, define the sensitivity in XGI as

$$
S=\frac{1}{2 \pi} \frac{\Delta \psi}{\Delta \alpha}
$$

i.e., the ratio between the change $\Delta \psi$ in fringe phase that is observed if the beam propagation angle changes by $\Delta \alpha$ (in the direction perpendicular to the grating lines), and that change of angle itself. In the case of an incident plane wave, this definition is equivalent to [33]

$$
S=\frac{D}{p_{2}} \stackrel{\text { Eq. }}{=} \frac{n p_{2}}{2 \lambda}
$$

It follows immediately from Equation 4 that the length of an interferometer with a given sensitivity $S$ is proportional to its grating pitch. 
Resolution in refraction angle. If we denote by $\sigma_{\alpha}$ the uncertainty in the determination of refraction angle resulting from the uncertainty $\sigma_{I}$ in the measurement of the image intensity, and define the reciprocal values of these quantities as the corresponding resolutions, then these two quantities are related by

$$
\sigma_{\alpha}^{-1} \propto \frac{I_{0}}{\sigma_{I}} \frac{v D}{p_{2}}=\frac{I_{0}}{\sigma_{I}} v S
$$

where $v$ is the fringe visibility and $I_{0}$ the average intensity over a phase-stepping scan or a moiré fringe. The resolution in refraction angle is thus proportional to the sensitivity.

Spatial resolution. The spatial resolution, i.e., the inverse size $\sigma_{x}^{-1}$ of the resolution element along the direction $x$ perpendicular to the grating lines, is limited by the diffraction of G1 to [6]

$$
\sigma_{x}^{-1}=\left(n p_{2}\right)^{-1}=\frac{2 \lambda}{S}
$$

Required coherence length. It follows immediately from Equations 2 and 4 that the required coherence length for $80 \%$ of the maximum visibility is

$$
\ell_{80 \%}=\lambda S
$$

i.e., the coherence length required depends only on the sensitivity, and not explicitly on the periods of the gratings used.

\section{CONCLUSIONS}

The considerations on grating properties show that there is no universally valid "best design" for a grating. In particular, the question whether a phase grating with $\pi$-shifting lines or one with $\pi / 2$ shift is better suited for a given device depends on the operating conditions of the device and on fabrication technology.

Equations 5 to 7 show that some of the most important performance characteristics and requirements of a grating interferometer have a simple relation to its sensitivity. Moreover, there is little influence of the grating pitch on many of these figures other than what is implicit in the sensitivity. However, even for fixed sensitivity, the intergrating distance $D$, and thus the length of the device, will depend on the grating pitch. In addition, high spatial resolution can only be efficiently achieved at small grating periods (although the sensitivity and angle resolution will also be limited because a small fractional Talbot order $n$ must be selected). These aspects favor small grating periods, despite the stricter requirements on mechanical precision and stability that follow.

\section{ACKNOWLEDGMENTS}

T. W. acknowledges support from the French research networks RTRA "Digiteo" and RTRA “Triangle de la Physique” (grants 2009-034T and 2009-79D). 


\section{REFERENCES}

1. C. David, B. Nöhammer, H. H. Solak, and E. Ziegler, Appl. Phys. Lett. 81, 3287-3289 (2002).

2. A. Momose, Jpn. J. Appl. Phys. 42, L866-L868 (2003).

3. T. Weitkamp, A. Diaz, B. Nöhammer, F. Pfeiffer, T. Rohbeck, P. Cloetens, M. Stampanoni, and C. David, Proc. SPIE 5535, 137-142 (2004).

4. A. Momose, S. Kawamoto, I. Koyama, and Y. Suzuki, Proc. SPIE 5535, 352-360 (2004).

5. T. Weitkamp, B. Nöhammer, A. Diaz, C. David, and E. Ziegler, Appl. Phys. Lett. 86, 054101 (2005).

6. T. Weitkamp, A. Diaz, C. David, F. Pfeiffer, M. Stampanoni, P. Cloetens, and E. Ziegler, Opt. Express 13, 6296-6304 (2005).

7. A. Momose, Jpn. J. Appl. Phys. 44, 6355-6367 (2005).

8. A. Momose, W. Yashiro, Y. Takeda, Y. Suzuki, and T. Hattori, Jpn. J. Appl. Phys. 45, 5254-5262 (2006).

9. T. H. Jensen, M. Bech, I. Zanette, T. Weitkamp, C. David, H. Deyhle, S. Rutishauser, E. Reznikova, J. Mohr, R. Feidenhans'1, and F. Pfeiffer, Phys. Rev. B 82, 214103 (2010).

10. W. Yashiro, Y. Terui, K. Kawabata, and A. Momose, Opt. Express 18, 16890-16901 (2010).

11. E. Ziegler, L. Peverini, I. V. Kozhevnikov, T. Weitkamp, and C. David, AIP Conf. Proc. 879, 778-781 (2007).

12. S. Rutishauser, I. Zanette, T. Weitkamp, T. Donath, and C. David, Appl. Phys. Lett. 99, 221104 (2011).

13. F. Pfeiffer, O. Bunk, C. Kottler, C. Grünzweig, C. David, M. Bech, G. Le Duc, A. Bravin, and P. Cloetens, Phys. Med. Biol. 52, 6923-6930 (2007).

14. T. Weitkamp, O. Bunk, F. Pfeiffer, C. David, J. Bruder, and P. Cloetens, Eur. J. Radiol. 68, S13-S17 (2008).

15. G. Schulz, T. Weitkamp, I. Zanette, F. Pfeiffer, F. Beckmann, C. David, S. Rutishauser, E. Reznikova, and B. Müller, J. R. Soc. Interface 7, 1665-1676 (2010).

16. I. Zanette, T. Weitkamp, S. Lang, M. Langer, J. Mohr, C. David, and J. Baruchel, Physica stat. sol. A 208, 2526-2532 (2011).

17. I. Zanette, M. Bech, F. Pfeiffer, and T. Weitkamp, Appl. Phys. Lett. 98, 094101 (2011).

18. S. A. McDonald, F. Marone, C. Hintermüller, G. Mikuljan, C. David, F. Pfeiffer, and M. Stampanoni, J. Synchrotron Radiat. 16, 562-572 (2009).

19. T. Weitkamp, I. Zanette, C. David, J. Baruchel, P. Bernard, M. Bech, H. Deyhle, T. Donath, J. Kenntner, S. Lang, J. Mohr, B. Müller, F. Pfeiffer, E. Reznikova, S. Rutishauser, G. Schulz, A. Tapfer, and J.-P. Valade, Proc. SPIE 7804, 780406 (2010).

20. V. Altapova, J. Butzer, T. dos Santos Rolo, P. Vagovic, A. Cecilia, J. Moosmann, J. Kenntner, J. Mohr, D. Pelliccia, V. F. Pichugin, and T. Baumbach, Nucl. Instrum. Meth. A 648 Suppl. 1, S42-S45 (2011).

21. F. Pfeiffer, T. Weitkamp, O. Bunk, and C. David, Nat. Phys. 2, 258-261 (2006).

22. J. F. Clauser, and S. F. Li, Phys. Rev. A 50, 2430-2433 (1994).

23. J. Herzen, F. Beckmann, T. Donath, M. Ogurreck, C. David, F. Pfeiffer, J. Mohr, E. Reznikova, S. Riekehr, A. Haibel, G. Schulz, and A. Schreyer, Proc. SPIE 7804, 780407 (2010).

24. T. Weitkamp, C. David, C. Kottler, O. Bunk, and F. Pfeiffer, Proc. SPIE 6318, 63180S.1-10 (2006).

25. J. Hartmann, Z. Instrumentenkd. 20, 47-58 (1900).

26. A. Olivo, and R. Speller, Appl. Phys. Lett. 91, 074106 (2007).

27. H. Wen, E. E. Bennett, M. M. Hegedus, and S. Rapacchi, Radiology 251, 910-918 (2009).

28. J. Primot, and N. Guérineau, Appl. Opt. 39, 5715-5720 (2000).

29. J. Rizzi, T. Weitkamp, N. Guérineau, M. Idir, P. Mercère, G. Druart, G. Vincent, P. da Silva, and J. Primot, Opt. Lett. 36, 1398-1400 (2011).

30. T. Weitkamp, P. Tafforeau, E. Boller, P. Cloetens, J.-P. Valade, P. Bernard, F. Peyrin, W. Ludwig, L. Helfen, and J. Baruchel, AIP Conf. Proc. 1221, 33-38 (2010).

31. C. David, J. Bruder, T. Rohbeck, C. Grünzweig, C. Kottler, A. Diaz, O. Bunk, and F. Pfeiffer, Microelectron. Eng. 84, 1172-1177 (2007).

32. E. Reznikova, J. Mohr, M. Börner, V. Nazmov, and P. J. Jakobs, Microsyst. Technol. 14, 1683-1688 (2008).

33. T. Donath, M. Chabior, F. Pfeiffer, O. Bunk, E. Reznikova, J. Mohr, E. Hempel, S. Popescu, M. Hoheisel, M. Schuster, J. Baumann, and C. David, J. Appl. Phys. 106, 054703 (2009). 